\title{
LA MOTIVACIÓN PROFESIONAL EN ESTUDIANTES DE PSICOLOGÍA
}

\section{PROFESSIONAL MOTIVATION IN PSYCHOLOGY STUDENTS}

Referencia del artículo

Castro Ascencio, M. de J. (2022). La motivación profesional en estudiantes de psicología. Revista Científica del Sistema de Estudios de Postgrado de la Universidad de San Carlos de Guatemala, 5(1), 1-7. DOI: https://doi.org/10.36958/sep.v5i1.90

\author{
Manuel de Jesús Castro Ascencio \\ manuel.castro@ues.edu.sv \\ https: / / orcid.org/0000-0003-4181-9883 \\ Doctorado en Educación con Especialidad en Educación Superior \\ Universidad de El Salvador
}

\section{RESUMEN}

OBJETIVO: reflexionar sobre la importancia de la motivación para la formación profesional de los estudiantes a nivel universitario, se sitúa en una línea de investigación de aprendizaje, educación y desarrollo en la educación superior. MÉTODO: se abordó desde un enfoque cualitativo con un diseño transversal, con alcance descriptivo, con fundamentación teórica y la sistematización de los resultados de una prueba piloto en una muestra de 25 estudiantes de primer año de psicología. RESULTADOS: en los resultados de la encuesta, se refleja que existe mayor predominancia del nivel medio de motivación profesional. En resultados del cuestionario, 12 estudiantes expresaron estudiar la carrera para ayudar a las personas. Mediante la observación participante, se constató manifestaciones de satisfacción y motivación, fuerte impulso a seguir estudiando, porque se les toma en cuenta, existe flexibilidad por parte del docente, se valoran sus opiniones y esfuerzos en el desarrollo de los contenidos. CONCLUSIÓN: se constató la importancia que tienen los planteamientos teóricos y empíricos en las manifestaciones motivacionales en el estudiante universitario. Así mismo, la elaboración de un diagnóstico motivacional, para constatar tanto el comportamiento de los diferentes indicadores evaluados, como las manifestaciones motivacionales de los estudiantes en las dimensiones cognitiva, afectiva-auto valorativa y formativa de la motivación profesional.

\section{PALABRAS CLAVE}

Motivación, motivación educativa y motivación profesional

\section{ABSTRACT}

OBJECTIVE: to reflect on the importance of motivation for the professional training of students at university level, is located in a line of research of learning, education and development in higher education. METHOD: was addressed from a qualitative approach with a transversal design, with descriptive scope, with theoretical foundation and the systematization of the results of a pilot test in a sample of 25 first-year students of psychology. RESULTS: in the results of the survey, it is reflected that there is a greater predominance of the average level of professional motivation. In the results of the questionnaire, 12 students expressed studying the career to help people. Through the participant observation, manifestations of satisfaction and motivation were observed, strong impulse to continue studying, because they are taken into account, there is flexibility on the part of the teacher, their opinions and efforts in the development of the contents are valued. CONCLUSION: the importance of theoretical and empirical approaches in motivational manifestations in university students was confirmed. Likewise, the elaboration of a motivational diagnosis, to verify both the behavior of the different indicators evaluated, as well as the motivational manifestations of the students in the cognitive, affective-self-valuative and formative dimensions of professional motivation.

\section{KEYWORDS}

Motivation, educational motivation and professional motivation 


\section{INTRODUCCIÓN}

El presente escrito acerca de la motivación profesional en estudiantes de psicología, tiene como objetivo reflexionar sobre la importancia de conocer el significado y la diferencia que existe entre la motivación como proceso psicológico, como proceso educativo y la motivación profesional, que tiene lugar en el nivel universitario, desde una perspectiva teórica y empírica. Se hacen los planteamientos teóricos de los autores que refieren la motivación como estado psicológico, como proceso educativo $\mathrm{y}$, por lo tanto, la motivación profesional, como también, algunos resultados de una reciente investigación en el contexto educativo y formativo de la motivación en estudiantes de Psicología. Para ello, el autor desarrolla los planteamientos teóricos referidos a la diferencia que existe entre la motivación como estado psicológico, motivación como herramienta eficaz del proceso educativo y la motivación profesional para fortalecer el nexo del sujeto con la carrera profesional que estudia. Así como también, se describen algunos resultados de la aplicación de los instrumentos en una prueba piloto. Seguidamente, se describen las conclusiones, en las cuales se explica la relación de la teoría y resultados principales obtenidos, mediante la triangulación de datos e información. Finalmente, se escriben las referencias que lo sustentan.

\section{MATERIALES Y MÉTODOS}

En la metodología se consideró el método dialéctico-materialista. Se abordó desde un enfoque cualitativo con un diseño transversal, con alcance descriptivo. Para ello, se asumieron los métodos teóricos, de análisis-síntesis, inducción-deducción, histórico-lógico y de modelación. De igual manera, los métodos empíricos y técnicas utilizadas fueron: la encuesta, cuestionario, entrevista, y la observación partici- pante, se describen resultados de una prueba piloto realizada con una muestra intencionada no paramétrica de veinticinco estudiantes de primer año de Psicología. Se hizo el análisis porcentual con el criterio de la triangulación de los datos e información obtenidos.

\section{RESULTADOS}

Para una fundamentación teórica de la motivación profesional, se desarrollan tres planteamientos que se describen a continuación:

Fundamentación teórica de la motivación como proceso psicológico

Herrera Soria y Zamora Guevara (2014) "La motivación es un proceso autoenergético de la persona que ejerce una atracción hacia un objetivo que supone una acción por parte del sujeto y permite aceptar el esfuerzo requerido para conseguirlo". Es decir, que la motivación es un proceso psicológico que impulsa energéticamente a la persona a lograr objetivos mediante esfuerzos propios que realiza. Por tanto, de esa energía impulsora depende, en gran medida el éxito de la actividad realizada. La energía que impulsa desde el interior al sujeto y que es un proceso psicológico necesario para toda actividad que se realice y que no debe faltar en el proceso de enseñanza-aprendizaje.

Fundamentación teórica de la motivación como proceso educativo

Polanco Hernández (2005) "Su polémica gira en torno a los factores que pueden influir y la diferencia entre el interés que se presenta por una tarea". Es decir, que la motivación despierta el interés y dirige las acciones que debe realizar un estudiante en lo conductual y cognitivo para alcanzar el éxito en su for- 
mación profesional. Mientras que, Gessa Gálvez (2007) considera "que no es solamente la parte afectiva que involucra, sino toda la personalidad la que se pone en acción ante la participación de la motivación". Es decir, que la motivación integra desde el componente afectivo, los componentes cognitivo y conductual y la de psicología del estudiante, para impulsarlo a la realización de sus actividades entre ellas el estudio.

Por tanto, Naranjo Pereira (2009) considera que "La motivación es un aspecto de enorme relevancia en las diversas áreas de la vida, entre ellas la educativa y la laboral". Es decir, que la motivación orienta al estudiante en las áreas educativa y laboral acompañando los objetivos que pretende lograr. Peña Estrada y Díaz (2015) "Para que un individuo realice una tarea deben concurrir tres requisitos: que el individuo pueda hacerlo (medios), que tenga conocimientos para hacerlo (habilidades y aptitudes) y que quiera hacerlo (motivación)". En tal sentido, deja claro que no se puede prescindir de la motivación, ya que sirve para promover esos conocimientos por parte del estudiante en su formación. Alemán Marichal y colaboradores (2018) considera que "La motivación por el estudio es un elemento consustancial a la labor educativa del profesor, que consiste en utilizar todas las vías posibles en el proceso de enseñanza-aprendizaje". Es decir que, si motivación es la clave para que una tarea se realice exitosamente, al estudiante se le facilita aprender mejor. Ya que no consiste solamente en tener los medios, las habilidades y las aptitudes, sino también la motivación que implica el querer hacer una tarea. Pero no una motivación como impulso inconsciente, sino como impulso en el contexto de la orientación consciente promovida por el profesor en las clases que desarrolla con sus estudiantes.
Fundamentación teórica de la motivación profesional en el nivel universitario

Ahora bien, la motivación profesional implica una estrecha relación del estudiante con la profesión que estudia, en la cual se unifican lo intrínseco con lo extrínseco. Así, Calderón (2010) considera que "las condiciones necesarias para su aparición radican en la unidad de lo cognitivo, referente a los intereses profesionales y lo afectivo, propio del impulso que como respuesta a las necesidades intrínsecas de la personalidad". Es decir que, estas condiciones tienen que ver con las etapas de la formación, desarrollo de intereses y habilidades profesionales, que lo impulsan a la consolidación de intereses, conocimientos y habilidades por parte del estudiante muy motivado con la profesión. Por tanto, se rompe con el modelo tradicional del proceso formativo inflexible, sino que se enfoca en un estilo dinámico del proceso de enseñanza-aprendizaje a nivel profesional.

Salabert Tortoló y colaboradores (2017) considera que "la motivación profesional se ubica en el nivel superior motivacional del sujeto, que dice: integra todo el conjunto de procesos psíquicos que mediatizan la estructuración de motivos de orientación hacia la futura profesión". Es decir, que el estudiante motivado puede profundizar con más facilidad en aquellos conocimientos de la profesión que ha decidido estudiar. Por lo que, la motivación interrelaciona y unifica lo intrínseco con lo extrínseco, interconecta los componentes cognitivo, afectivo y conductual de la psicología del estudiante.

En tal sentido, en la tabla 1 se muestran los resultados obtenidos de la aplicación de una encuesta aplicada a 25 estudiantes del primer año de Psicología. 
Tabla 1. Indicadores con niveles de motivación profesional con porcentajes

\begin{tabular}{|c|c|c|c|c|c|c|c|}
\hline No. & Indicadores/Niveles con \% & Bajo & $\%$ & Medio & $\%$ & Alto & $\%$ \\
\hline 1 & Nivel de conocimientos & 4 & 16.00 & 20 & 80.00 & 1 & 4.00 \\
\hline 2 & Desarrollo de habilidades & 2 & 8.00 & 19 & 76.00 & 4 & 16.00 \\
\hline 3 & Experiencias adquiridas & 2 & 8.00 & 17 & 68.00 & 6 & 24.00 \\
\hline 4 & Intereses, aptitudes y objetivos & 1 & 4.00 & 17 & 68.00 & 7 & 28.00 \\
\hline 5 & Aspiraciones y convicciones & 2 & 8.00 & 15 & 60.00 & 8 & 32.00 \\
\hline 6 & Nivel de pretensiones & 0 & - & 12 & 48.00 & 13 & 52.00 \\
\hline 7 & Toma de decisiones & 1 & 4.00 & 8 & 15.00 & 16 & 64.00 \\
\hline 8 & Capacidad de reflexión & 1 & 4.00 & 11 & 44.00 & 13 & 52.00 \\
\hline 9 & Capacidad para valorar & 1 & 4.00 & 5 & 20.00 & 19 & 76.00 \\
\hline 10 & Dominio de los aprendizajes & 0 & - & 17 & 68.00 & 8 & 32.00 \\
\hline
\end{tabular}

Fuente: Castro (2019). Información de base de datos del estudio de campo.

La tabla muestra el listado de indicadores, el número de sujetos y el porcentaje que corresponden al nivel bajo, medio y alto de la motivación profesional.

Refleja mayor predominancia del nivel medio con un $80 \%$ de los sujetos en el nivel medio de conocimientos, un $76 \%$ en el desarrollo de habilidades, un $68 \%$ en experiencias adquiridas, en intereses, aptitudes y objetivos por parte de los estudiantes hacia la profesión de Psicología.

En un $60 \%$ aspiraciones y convicciones. A diferencia, se encontró que existe nivel alto con un $76 \%$ de los sujetos en capacidad para valorar y con $64 \%$ en la toma de decisiones.
A continuación, se presente la figura 1 en la que se muestran los niveles de motivación por parte de los sujetos con base a los puntajes obtenidos de la aplicación de la encuesta. 
Figura 1. Sujetos que se representan en niveles de motivación respecto a los indicadores

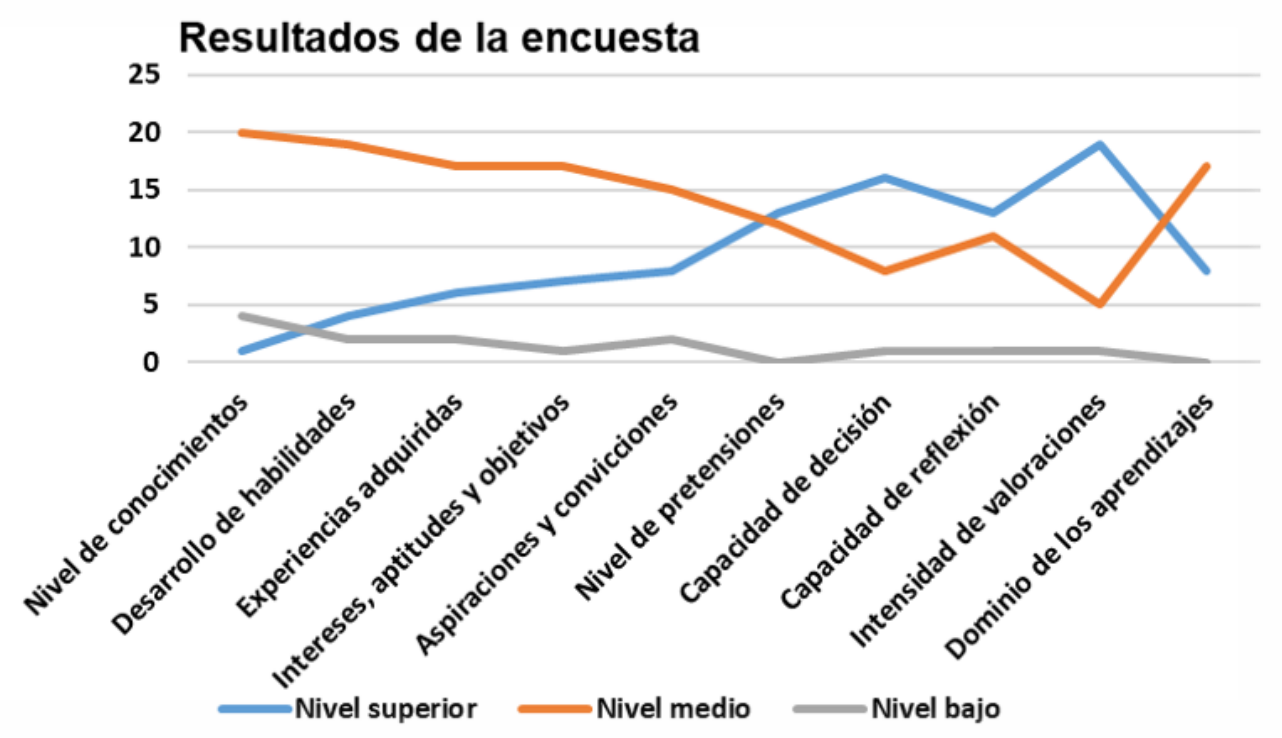

Fuente: Castro (2019). Información de base de datos del estudio.

La figura refleja que 20 sujetos se ubican en el nivel medio de motivación, de manera particular en los indicadores de conocimientos, desarrollo de habilidades y dominio de los aprendizajes. El nivel superior 19 sujetos reflejan la intensidad de las valoraciones y 16 sujetos la capacidad de decisión por estudiar la carrera.

Por otra parte, en los resultados del cuestionario en la dimensión cognitiva, afectiva-auto valorativa: 13 sujetos expresan estudiar la profesión para poder ayudar a las personas, un sujeto menciona que estudia para conocer las conductas de las personas, dos sujetos manifiestan el interés por adquirir conocimientos, un sujeto menciona que lo motivan los docentes, la familia y su convicción, un sujeto dice tener ganas de superarse, a otro sujeto le gusta la carrera, a diferencia de otro que estudia la profesión para desarrollarse en el ámbito laboral; un sujeto quiere ser profesional para tener donde vivir, otro quiere especializarse en psicología forense; finalmente, un sujeto considera que es la carrera que tiene futuro por su importancia.

De la misma manera, en la dimensión afectiva auto-valorativa, los estudiantes expresaron estar motivados en estudiar la carrera para ayudar a las personas y adquirir conocimientos sobre la profesión. Así mismo, los estudiantes afirman desconocer el significado de autodeterminación profesional y responden de acuerdo a su propia interpretación. Sin embargo, otros estudiantes declararon tener el deseo de terminar sus estudios y ejercer la profesión, ser mejor cada día, ser alguien en la vida, ganar dinero, ayudar a los demás con sus problemas mentales y brindar buen trato a las personas.

En contraste, en la entrevista, los estudiantes expresaron sentirse satisfechos y motivados, lo que les impulsa a seguir estudiando, ya que se les toma en cuenta, existe flexibi- 
lidad por parte del docente, se valoran sus opiniones y esfuerzos en el desarrollo de los contenidos. Por consiguiente, en la observación participante, se constató poca motivación, escasa participación y se mostraban desinteresados en el desarrollo de los contenidos en las clases, cuando no se fomentaba el trabajo en equipo e interactivo.

\section{DISCUSIÓN}

La construcción teórica y empírica de la motivación profesional permitió plantear las manifestaciones motivacionales que se reflejan en el estudiante universitario. Así, en un diagnóstico motivacional de una prueba piloto, se constató el comportamiento de los indicadores de las dimensiones cognitiva, afectiva-autovalorativa y formativa de la motivación profesional. Por consiguiente, en la encuesta, los resultados reflejaron un nivel medio de motivación en cuanto a conocimientos, desarrollo de habilidades, experiencias e intereses. Así, en el cuestionario los estudiantes opinaron que estudian para ayudar a las personas; de igual modo, en la entrevista y la observación participante, los estudiantes expresan estar satisfechos y motivados, porque se toma en cuenta sus opiniones, se les hace trabajar en equipo y se valoran sus esfuerzos.

\section{REFERENCIAS}

Alemán Marichal, B., Navarro de Armas, O. L., Suárez Díaz, R. M., Izquierdo Barceló, Y. y Encinas Alemán, T. de la C., (2018). La motivación en el contexto del proceso enseñanza-aprendizaje en carreras de las Ciencias Médicas, Revista Médica Electrónica, vol.40 no.4 Matanzas. http: //scielo.sld.cu > scielo
Calderón, C. V. (2010). Etapas de la orientación profesional desde un enfoque integrador, Centro Mixto "Juan George Soto Cuesta" Bajada de Rondón. Holguín, Cuba. https://www.eumed.net/rev/tlatemoani/06/ctf.htm

Gessa Gálvez, M. M. (2007). Estrategia educativa para la motivación profesional de los estudiantes que ingresan en la carrera de Agronomía en la Facultad de Montaña. Facultad de Educación y Psicología, Universidad Girona. https://www. tesisenred.net > https://dialnet.unirioja.es/servlet/tesis?codigo $=89158$

Herrera Soria, J., Zamora Guevara, N. (2014). ¿Sabemos realmente que es la motivación?, correo Científico Médico, vol. 18 no. 1 Holguin. http: / /scielo.sld.cu > scielo

Naranjo Pereira, M. L. (2009). Motivación: perspectivas teóricas y algunas consideraciones de su importancia en el ámbito educativo, Revista Educación 33(2), 153-170, ISSN: 0379-7082, Universidad de Costa Rica, Costa Rica. https://doi. org $/ 10.15517 /$ revedu.v33i2.510

Peña Estrada, C. \& Díaz, M. J. (2015). La motivación laboral como herramienta de gestión en las organizaciones empresariales. 96. Repositorio de la Universidad Pontificia Comillas. https://repositorio.comillas.edu/jspui/ bitstream/11531/4152/1/TFG001138. pdf. https://repositorio.comillas.edu , TFG001138

Polanco Hernández, A. (2005). La motivación en los estudiantes universitarios, Revista electrónica "Actualidades Investigativas en Educación", Universidad de Costa Rica, San Pedro de Montes de Oca. https://doi.org/10.15517/aie.v5i2.9157

Salabert Tortoló, I., Naipe Delgado, M. C., Garriga, N., Alfonso Prínce, J. C., Dihigo Faz, M. T., y Núñez Valdés, L. (2017). La motivación profesional en los estudian- 
tes de las Ciencias Médicas. Revista Médica Electrónica, 39(3), 630-639. Cuba, Universidad de Ciencias Médicas de Matanzas http: / /www.revmedicaelectronica.sld.cu/index.php/rme/article/viewFile/1989/pdf_268

\section{SOBRE EL AUTOR}

\section{Manuel de Jesús Castro Ascencio}

Licenciado en Psicología, Maestría en Métodos y Técnicas de Investigación Social, actual doctorando del Doctorado en Educación, con Especialidad en Educación Superior. En la actualidad ejerce la docencia como profesor en la sección de Psicología de la Facultad Multidisciplinaria Oriental, Universidad de El Salvador.

\author{
Conflicto de intereses \\ Declara no tener ningún conflicto de intereses. \\ Declaración de consentimiento informado \\ El estudio se realizó, respetando el Código de ética y buenas prácticas editoriales de publicación. \\ Derechos de uso \\ Copyright $\odot 2022$ por Manuel de Jesús Castro Ascencio \\ Este texto está protegido por una licencia Creative Commons 4.0. Internacional
}

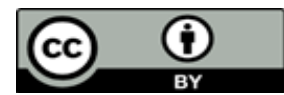

Usted es libre para compartir, copiar y redistribuir el material en cualquier medio o formato y adaptar el documento, remezclar, transformar y crear a partir del material para cualquier propósito, incluso comercialmente, siempre que cumpla la condición de atribución: usted debe reconocer el crédito de una obra de manera adecuada, proporcionar un enlace a la licencia, e indicar si se han realizado cambios. Puede hacerlo en cualquier forma razonable, pero no de forma tal que sugiera que tiene el apoyo del licenciante o lo recibe por el uso que hace. 